Gut and Liver, Vol. 10, No. 1, January 2016, pp. 1-2

\title{
Proton Pump Inhibitor-Responsive Esophageal Eosinophilia: Controversies and Its Clinical Implications
}

\author{
Cheol Min Shin and Dong Ho Lee \\ Department of Internal Medicine, Seoul National University Bundang Hospital, Seongnam, Korea
}

See "Clinicopathologic Analysis of Proton Pump Inhibitor-Responsive Esophageal Eosinophilia in Korean Patients" by Da Hyun Jung, et al. on page 37, Vol. 10. No. 1, 2016

The definition of esophageal eosinophilia has changed over the past years. ${ }^{1}$ Before 1980s, esophageal eosinophilia had been merely regarded as a manifestation of gastroesophageal reflux disease (GERD). Since the mid-1990s, there have been numerous studies that reported esophageal eosinophilia with symptoms other than GERD, characterized by esophageal motility disorders and/or food intolerance, both in children and young adults. Later on, these patients were categorized into eosinophilic esophagitis (EoE). EoE is a disorder that is characterized clinically by symptoms related to esophageal dysfunction and histologically by an eosinophil-rich inflammation that is limited to the esophagus. ${ }^{2}$

According to the current guidelines, it is recommended to rule out other disorders associated with similar clinical, histological, or endoscopic features, especially GERD, using a 4- to 8-week high-dose proton pump inhibitor (PPI) trial and/or a $\mathrm{pH}$ monitoring before a formal diagnosis of EoE, because of the nonspecific nature of esophageal eosinophilia. ${ }^{2}$ Thus the patients with esophageal eosinophilia that responds to PPI therapy are not considered to have EoE. Nonetheless, several case studies caused confusions that reported the patients with endoscopic and symptomatic features of EoE who showed resolution of eosinophilia with PPI therapy alone..$^{3}$ It led to the necessity to define a third disorder, PPI-responsive esophageal eosinophilia (PPI-REE).

It is unclear whether PPI-REE represents an independent clinical disorder, or either an EoE-variant or a GERD variant. According to a recent meta-analysis, the response rate of PPI therapy in symptomatic esophageal eosinophilia was $60.8 \%$ as clinical response, 50.5\% as histologic remission. ${ }^{4}$ But it is unclear whether these patients with esophageal eosinophilia had
PPI-REE or GERD based on PPI response only. In addition, it is impossible to differentiate EoE from PPI-REE by clinical or histopathologic findings before PPI treatment. ${ }^{5}$ As a matter of fact, it is still controversial whether PPI-REE is a distinct disease entity or not. PPI-REE might be a transient condition because some patients who had an initial response to PPI therapy became resistant to PPI on long-term follow-up. ${ }^{6}$ This makes it difficult to understand the pathophysiology of PPI-REE.

The symptomatic and histologic responses to PPI treatment have been attributed to several mechanisms. First, some patients with PPI-REE may have concomitant GERD. In fact, GERD is common in the general population, so acid suppression may attenuate some of the patient's symptoms and/or eosinophilic infiltration in the patients with both PPI-REE and GERD. Second, PPI had an effect on esophageal permeability which affects the esophageal antigen exposure. Previously, it has been reported that ultrastructural alternations (i.e., dilation of intercellular spaces) induced by GERD could be reversible by PPI treatment, followed by the relief of reflux symptoms. ${ }^{7}$ One recent study reported that PPIs could partially restore esophageal mucosal integrity in patients with PPI-REE but not in those with EoE. ${ }^{8}$ Third, it is possible that PPIs may have anti-inflammatory functions independent of acid suppression. PPIs can act as antioxidants, directly inhibit neutrophils and monocytes, which decrease the expression of proinflammatory cytokines. ${ }^{9}$

Previous studies on PPI-REE have been performed mainly in Western countries, but there has been only limited evidence in Asian population, mainly originating from Japan. Recently, Jung et al. ${ }^{10}$ reported the clinicopathologic characteristics in the six Korean PPI-REE patients. In spite of the limitations such as

\footnotetext{
Correspondence to: Dong Ho Lee

Department of Internal Medicine, Seoul National University Bundang Hospital, 82 Gumi-ro 173beon-gil, Bundang-gu, Seongnam 13620, Korea Tel: +82-31-787-7006, Fax: +82-31-787-4051, E-mail: dhljohn@snubh.org pISSN 1976-2283 eISSN 2005-1212 http://dx.doi.org/10.5009/gnl15601
} 
small sample size, it was the first Korean study focusing on PPIREE. In this study, allergic tests such as serum IgE level, skin prick tests, endoscopy and histopathologic analysis including eotain-3 were comprehensively reviewed. The authors concluded that the clinicopathologic characteristics were similar to those in the Western studies. In summary, further study is necessary with respect to the pathophysiology of esophageal eosinophilia, and also prospective long-term follow-up study is warranted for the patients with PPI-REE treated with PPI in Asian population.

\section{CONFLICTS OF INTEREST}

No potential conflict of interest relevant to this article was reported.

\section{REFERENCES}

1. Furuta GT, Katzka DA. Eosinophilic esophagitis. N Engl J Med 2015;373:1640-1648.

2. Liacouras CA, Furuta GT, Hirano I, et al. Eosinophilic esophagitis: updated consensus recommendations for children and adults. J Allergy Clin Immunol 2011;128:3-20.e6.

3. Ngo P, Furuta GT, Antonioli DA, Fox VL. Eosinophils in the esophagus: peptic or allergic eosinophilic esophagitis? Case series of three patients with esophageal eosinophilia. Am J Gastroenterol 2006;101:1666-1670.
4. Lucendo AJ, Arias Á, Molina-Infante J. Efficacy of proton pump inhibitor drugs for inducing clinical and histologic remission in patients with symptomatic esophageal eosinophilia: a systematic review and meta-analysis. Clin Gastroenterol Hepatol 2016;14:1322.e1.

5. Dellon ES, Speck 0, Woodward K, et al. Markers of eosinophilic inflammation for diagnosis of eosinophilic esophagitis and proton pump inhibitor-responsive esophageal eosinophilia: a prospective study. Clin Gastroenterol Hepatol 2014;12:2015-2022.

6. Dohil R, Newbury R0, Aceves S. Transient PPI responsive esophageal eosinophilia may be a clinical sub-phenotype of pediatric eosinophilic esophagitis. Dig Dis Sci 2012;57:1413-1419.

7. Calabrese C, Bortolotti M, Fabbri A, et al. Reversibility of GERD ultrastructural alterations and relief of symptoms after omeprazole treatment. Am J Gastroenterol 2005;100:537-542.

8. van Rhijn BD, Weijenborg PW, Verheij J, et al. Proton pump inhibitors partially restore mucosal integrity in patients with proton pump inhibitor-responsive esophageal eosinophilia but not eosinophilic esophagitis. Clin Gastroenterol Hepatol 2014;12:18151823.e2.

9. Cheng E, Zhang X, Huo X, et al. Omeprazole blocks eotaxin-3 expression by oesophageal squamous cells from patients with eosinophilic oesophagitis and GORD. Gut 2013;62:824-832.

10. Jung DH, Yun GW, Lee YJ, Jo Y, Park H. Clinicopathologic analysis of proton pump inhibitor-responsive esophageal eosinophilia in Korean patients. Gut Liver 2016;10:37-41. 